\title{
The Role of Substrate Corrugation in Helium Monolayer Solidification
}

\author{
M. E. Pierce and E. Manousakis \\ Department of Physics and Center for Materials Research and Technology, Florida State University, Tallahassee, FL \\ $32306-4350$
}

(February 26, 2018)

\begin{abstract}
We investigate the first layer of helium adsorbed on graphite with path-integral Monte Carlo, examining the role of substrate corrugations on the phase diagram. When no corrugations are present, the equilibrium state of the system is a liquid phase, with solidification occurring only under compression but before layer promotion. We determine the solid-liquid coexistence region and compare our results to recent Green's function Monte Carlo calculations on the same system. When substrate corrugations are included, we find that the equilibrium phase is the $\sqrt{3} \times \sqrt{3}$ commensurate solid phase that is well known from experiment. The melting behavior, heat capacity, and single particle binding energy are determined and compared to experiment. We further find that for densities below the commensurate coverage, the low temperature phase of the system consists of solid clusters in coexistence with coalesced vacancies. We find no first layer liquid phase and so no superfluidity in this layer, in contrast to some rather recent suggestions.
\end{abstract}

PACS numbers 67.70.+n, $67.40 \mathrm{Kh}$

\section{INTRODUCTION}

Quantum films such as helium adsorbed on graphite surface are characterized by a rich phase diagram as one varies the number of the deposited helium atoms. As a function of the helium coverage, the helium film grows in atomically thin layerst 3 and at least seven such layers may be clearly distinguished and studied on a wellprepared substrate.

During the last several years, extensive heat capacity measurements 46 of the first six layers have been performed, and superfluidity in the higher layers has been detected by both torsional oscillatord $\mathrm{B}$ and third sound measurements. More specifically, the first layer of helium adsorbed on graphite has been the laboratory for study of a variety of phenomena. In this layer a $\sqrt{3} \times \sqrt{3}$ commensurate solid phase forms in which one-third of the available substrate adsorption sites are occupied. 4 (6) 10 For densities above this commensurate density, the monolayer is characterized by a region of domain wall phases and at even higher monolayer densities it forms an incommensurate triangular solid phase. 1123 The phase diagram below the commensurate density at low temperatures is not well understood and there are two competing scenarios. In one scenario this region corresponds to a solid with clustered vacancies.14 According to this picture, since the commensurate solid phase is in the same universality class as the three-state Potts model, 120 at lower densities the film should consist of a commensurate solid with vacancies. When the temperature is raised, the solid melts continuously. Lowering the temperature causes the vacancies to coalesce, which is a first order transition. The difference between the temperatures of these two transitions becomes smaller as the density is lowered until they meet at a tricritical point.

The second scenario for the low density region of the phase diagram of the sub-monolazer was suggested more recently by Greywall and Busch that their measured heat capacity is not linear in density for the entire region below the commensurate density, as it must be for phase coexistence. Thus, they propose that a self-bound liquid phase occurs at about 0.04 atom/ $\AA^{2}$. This conclusion is supported by $2 \mathrm{D}$ tariational calculations.20 However, direct measurements 1.21 detect no superfluidity, possibly because of poor substrate connectivity.

In a previous publication 22, we have used the pathintegral Monte Carlo method and realistic helium-helium and helium graphite interactions to study the monolayer at or below the commensurate density. We found that the presence of corrugations, which causes the commensurate solid at $1 / 3$ coverage, creates solid commensurate clusters at densities below the commensurate density. We found that it is the melting of these monolayer clusters which gives rise to a specific heat maximum which was incorrectly interpreted 3 as onset of monolayer superfluidity. In this paper we examine in detail the role of substrate corrugation on the first layer phase diagram using the path-integral Monte Carlo method. First, we present results for helium adsorbed on a smooth substrate. The helium-graphite interaction is basedon the laterally averaged potential of Carlos and Cole.23 This layer exhibits liquid and solid phases, and we calculate the phase diagram at low temperatures. These results are compared with recent Green's function Monte Carlo calculations.24 The second layer promotion density is also determined. Next, calculations including substrate corrugations are discussed, and direct comparisons of the melting behavior, specific heat, and single particle binding energy that we obtain are made with experiment. Finally, having verified our method by the above comparisons, we examine the low density, low temperature phase of the helium monolayer. We determine that for all coverages below the commensurate density, the system consists of solid clusters in phase coexistence with coalesced vacancies. No liquid phase occurs and so there is no possibility for 
first layer superfluidity.

\section{SIMULATION METHOD}

Our study employs a path-integral Monte Carlo (PIMC) method for simulating bose systems. Details on the application of this method to bulk helium can be found in the review by Ceperley,25 and our modifications for the simulation of adsorbed helium films may be found in a previous publication.26 We briefly review the procedure now in order to explain the calculations presented in this paper.

PIMC evaluates properties of an $N$-body quantum system at the inverse temperature $\tau$ by sampling the partition function $Z$. $Z$ is expanded as a path-integral by inserting $M$ intermediate configurations:

$$
\begin{aligned}
Z= & \frac{1}{N !} \sum_{P} \int \ldots \int d^{3} R_{1} \ldots d^{3} R_{M} d^{3} R \\
& \times \rho\left(\mathbf{R}_{1}, \mathbf{R}_{2} ; \tau\right) \rho\left(\mathbf{R}_{2}, \mathbf{R}_{3} ; \tau\right) \ldots \rho\left(\mathbf{R}_{M}, P \mathbf{R}_{1} ; \tau\right),
\end{aligned}
$$

where $\rho$ is the density matrix, $\mathbf{R}_{i}$ is a configuration of $N$ particles, and $\tau=\beta / M$. By taking $M$ large enough, the density matrices at $\tau$ can be accurately approximated. The particular PIMC method that we employ ergodically samples both particle positions and particle permutations.

The key element needed in the first layer simulation is an accurate approximation for the high temperature density matrices. For bulk helium and helium on a smooth substrate, the starting approximation for the density matrix can be taken with a temperature as low as $40 \mathrm{~K}$. In this paper, results for the first layer on a smooth substrate were obtained using the same starting approximation for the density matrix thatwas used in previous calculations for the second layer.26

However, as discussed in our previous publication 26 a helium-graphite interaction that includes substrate corrugations makes the starting approximation used for smooth substrates impractical, and so we must use a simpler form. For these calculations, we instead use the high temperature expansion of the density matrix. At sufficiently small values of the inverse temperature, the density matrix is given by the Trotter approximation:

$$
\exp (-\tau \hat{H}) \approx \exp (-\tau \hat{T}) \exp (-\tau \hat{V})
$$

where $\hat{H}, \hat{T}$ and $\hat{V}$ are the Hamiltonian and the kinetic and potential energy operators, respectively, and $\tau$ is the inverse temperature. This allows us to approximate the density matrices at $\tau$ as

$$
\begin{aligned}
\rho\left(\mathbf{R}, \mathbf{R}^{\prime} ; \tau\right) \propto & \exp \left[-\pi\left(\mathbf{R}_{i}-\mathbf{R}_{i+1}\right) / \lambda_{T}^{2}\right. \\
& \left.-\tau\left(V\left(\mathbf{R}_{i}\right)+V\left(\mathbf{R}_{i+1}\right)\right) / 2\right]
\end{aligned}
$$

where $R$ and $R^{\prime}$ are the position vectors for $N$ particle configurations, and $\lambda_{T}$ is the thermal wavelength. This is sometimes referred to as the semiclassical approximation, and is accurate for sufficiently high starting temperatures. Averaging over the potential energy terms is referred to as the endpoint approximation. The potential energy term $V\left(\mathbf{R}_{i}\right)$ is the sum of all helium-helium and helium-graphite interactions. The exponent of Eq. 3 is the first term in an expansion in powers of $\tau 27$

In the semiclassical calculations, we have used $200 \mathrm{~K}$ as the starting temperature, meaning that 200 inversetemperature slices are required to reach $1 \mathrm{~K}$. We have verified that this temperature is sufficiently high for the approximation to be accurate by comparing the energy calculated at $4 \mathrm{~K}$ using $200 \mathrm{~K}$ and $320 \mathrm{~K}$ as the starting temperatures. The energy values obtained agreed within error bars. We have also adopted a three-level bisection, $l=3$, for sampling the positions. We verified that this level gave a lower energy at $4 \mathrm{~K}$ than calculations using $l=2$ and $l=4$. The acceptance rate using $l=3$ is approximately $40 \%$. We have further verified that the density matrix is well approximated at $200 \mathrm{~K}$ by Eq. 3 by including the next term in the series expansion in powers of $\tau$. Energy values calculated with this starting approximation agreed within error bars with those that used Eq. 3.

We did find that higher order terms in $\tau$ must be included in the energy estimator. The values we report in Sec. IV are obtained using

$$
<E>=\frac{3 N}{2 \tau}+<T>+<V>+\tau^{2} \hbar^{2} /(8 m)<(\nabla V)^{2}>
$$

for the energy expectation value, where $N$ is the number of particles, $\langle T\rangle$ and $\langle V\rangle$ are the kinetic and potential energy expectation values, and $m$ is the mass of the helium atom. Note that in PIMC the effective action, defined as the natural log of the density matrix, not the energy estimator, is used to choose between configurations in the Monte Carlo procedure.

Once the machinery for the Monte Carlo method is in place, helium-helium and helium-substrate potentials are required for input. We use the Azid28 potential for the helium-helium interaction. For the helium-graphite interaction, we have adopted the anisotropicLennard-Jones potential proposed by Carlos and Cole.23 This potential can be expressed as a Fourier series in the reciprocal lattice vectors, $\mathbf{G}$, of the graphite substrate. In cylindrical coordinates $(\rho, \mathrm{z})$ the expansion is

$$
V(\mathbf{r})=V_{0}(z)+\sum_{\mathbf{G}} V_{\mathbf{G}}(z) \exp (i \mathbf{G} \cdot \rho),
$$

where $V_{0}(z)$ is the laterally averaged potential, and $V_{\mathbf{G}}(z)$ gives the corrugation strength. The mathematical forms for these terms are given elsewhere 23 and the series converges rapidly. For the smooth substrate, we use only $V_{0}(z)$. For calculations that included corrugations, we kept the six lowest, equivalent values of $\mathbf{G}$ in the expansion. 
The limitation of our method is thus related to the accuracy of the potentials available to us. It is possible, for instance, that the substrate may substantially mediate the helium-helium interaction.29 This is the so-called McLachlan interaction 30 . We have performed calculations both with and without this term. Another possible concern is the helium-graphite potential, Eq. 5, which may overestimate the corrugation strength. Lowering the corrugation height will effect the properties of the first layer, with the favored phase becoming liquid instead of solid at sufficiently small corrugations. We have examined this effect by repeating some of the calculations with the corrugation strength set to $50 \%$ and to $75 \%$ of the value obtained from the Carlos-Cole model. Details of these tests of the limits of our interaction model are given below.

Particle permutations were also included in the calculations and were observed in the film on the smooth substrate at low densities. However, we have found that permutations do not play a role in the first layer on the corrugated substrate. We have allowed for permutations at intermediate densities for temperatures as low as 0.571 $\mathrm{K}$ with the level of the path bisectioning taken as large as 5 (32 beads updated in one move) but never observed any particle exchanges. This has been checked in calculations both with and without the McLachlan interaction.

\section{RESULTS FOR SMOOTH GRAPHITE SURFACE}

\section{A. Energy Calculations}

In this section we report simulation results for the first layer obtained by using only the laterally averaged portion $V_{0}(z)$ of the helium-graphite potential. We ignore substrate corrugations. This system has recently been studied with the Green's Function Monte Carlo (GFMC) method,24 so we have the opportunity to verify that the full implementation of our method is in agreement with the results of these complementary calculations. The GFMC calculations employ the same helium-graphite potential as we do but use an older form of the heliumhelium potential 31 The older form was used since the authors wanted to make direct comparisons with previous work on two-dimensional helium using this potential. 32 The newer potential that we use for the helium-helium interaction is somewhat more attractive (the well depth is about $0.1 \mathrm{~K}$ greater), so we expect the energy per particle to be somewhat lower near the equilibrium density. This has been observed in recent zero-temperature calculations for two dimensional liquid helium that employ the newer potential. 33

Guided by previous simulations, we expect the helium film to have a self-bound liquid phase and to solidify at high densities, before promotion to the next layer occurs. Calculations at low and intermediate densities are per- formed using a square simulation cell, while solid phase calculations use a rectangular simulation cell that accommodates the periodic structure of the triangular solid. It is not necessary in PIMC to employ different forms for the density matrix for the liquid and solid phases. Liquid calculations employed 16 particles, while solid phase calculations used 30 particles. Both sets of calculations were performed at $400 \mathrm{mK}$. Calculations at $500 \mathrm{mK}$ are in agreement with these results, indicating that we have converged to the zero-temperature limit. We verified that there were no finite-size errors in the liquid phase by repeating some of the calculations with 32 particles. The energy values at the two temperatures were in agreement. Finite size errors were found to be negligible for the solid phase also, since the energy value calculated at 0.0689 atom $/ \AA^{2}$ using the rectangular simulation cell (30 particles) agreed with the value calculated using the square simulation cell (16 particles).

These calculations are somewhat different from those we discuss in the other section of this paper. The potential between the active helium atoms and the underlying substrate is featureless in the plane of the substrate, so the size of the simulation cell may be varied continuously. This allows us to keep the number of particles constant for each phase. In contrast, other calculations we have performed (including those with substrate corrugations taken into account) were with a varying number of particles and a constant simulation cell size. The two methods lead to somewhat different forms for the Maxwell construction. In the present case, liquid-solid coexistence will be characterized by a linear region in the dependence of the energy per particle on the atomic area (inverse volume).

Figure 11 displays our results for the energy of the first layer liquid. Also shown are the results obtained from GFMC. For all points except the near equilibrium, the two calculations agree within error bars. Near the energy minimum, there is some disagreement, but even here the computed values differ only by about $0.6 \%$. This is perhaps attributable to the different helium-helium potentials used in the two calculations. Figure 2 shows our results and the GFMC results for the first layer solid phase. Again, there is excellent agreement between the two methods.

Following the typical procedure, we have determined the equations of state for the liquid and solid phases by fitting our energy values to the polynomials

$$
\begin{aligned}
& E / N=e_{0}+B\left(\frac{\rho-\rho_{0}}{\rho_{0}}\right)^{2}+C\left(\frac{\rho-\rho_{0}}{\rho_{0}}\right)^{3}, \\
& E / N=\alpha+\beta \rho+\gamma \rho^{2}+\delta \rho^{3} .
\end{aligned}
$$

for the liquid and solid phases, respectively. Values for the fitted parameters are given in Table Errors for the liquid phase are indicated. The values shown for the solid phase were used in the plot of Fig. 2. The values given for $\beta, \gamma$, and $\delta$ are accurate to one significant figure.

For the liquid phase, the parameters $e_{0}$ and $\rho_{0}$ are 


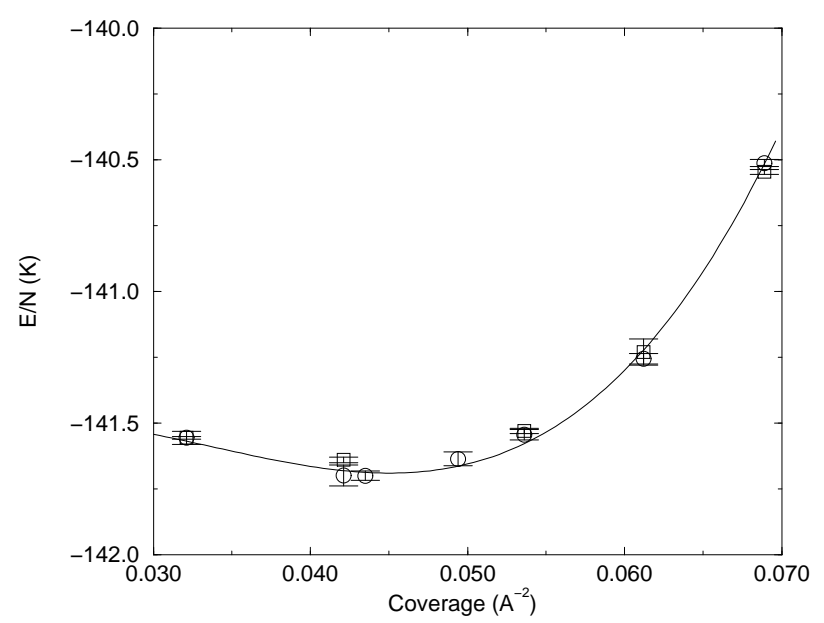

FIG. 1. Energy values for the first layer liquid. The circles are our results obtained with PIMC. The squares are GFMC results. 24 The line is a least squares fit of the polynomial, Eq. (7) to our data.

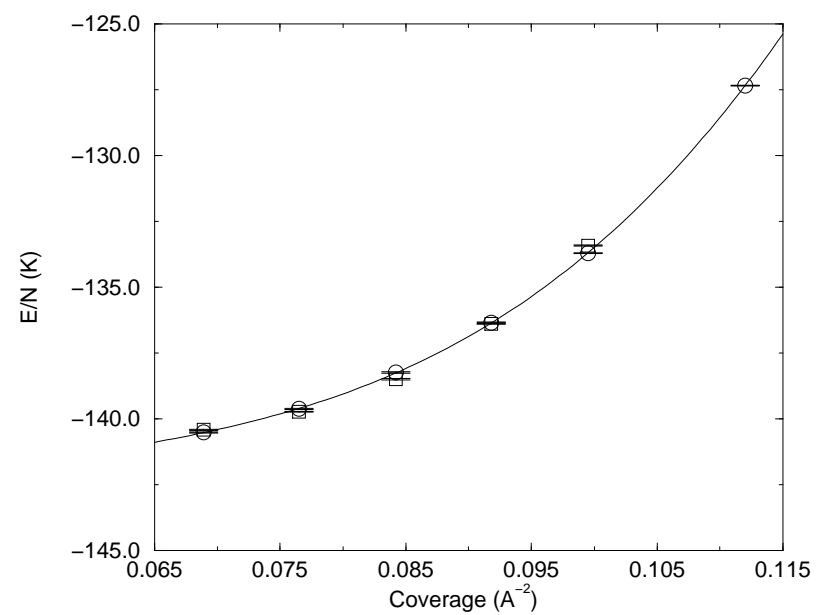

FIG. 2. Energy values for the first layer triangular solid. The symbols have the same meaning as in Fig. 2. The line is determined from the fit of Eq. (7) to our data.

\begin{tabular}{|lr|lr}
\hline \hline Parameter & Liquid & Parameter & Solid \\
\hline $\mathrm{e}_{0}(\mathrm{~K})$ & $-141.689(14)$ & $\alpha(\mathrm{K})$ & -157.46 \\
$\rho_{0}\left(\AA^{-2}\right)$ & $0.0450(06)$ & $\beta\left(\mathrm{K} / \AA^{2}\right)$ & -679.83 \\
$\mathrm{~B}(\mathrm{~K})$ & $2.42(27)$ & $\gamma\left(\mathrm{K} / \AA^{4}\right)$ & -10504.77 \\
$\mathrm{C}(\mathrm{K})$ & $3.29(67)$ & $\delta\left(\mathrm{K} / \AA^{6}\right)$ & 61032.24 \\
$\chi^{2} / \nu$ & 2.57 & $\chi^{2} / \nu$ & 1.3 \\
\hline \hline
\end{tabular}

TABLE I. Fitted parameters for the liquid and solid equations of state. The errors in the last digits of the parameters for the liquid phase are given in parenthesis.

the equilibrium energy per particle and the equilibrium coverage. The equilibrium density we obtain, 0.0450 atom $/ \AA^{2}$, is in reasonable agreement with the GFMC value of 0.0443 . Below this density, the system enters gas-liquid coexistence in the thermodynamic limit. In simulation, phase separation will not occur immediately because of the finite cost of creating the phase boundary. The system will instead enter a "stretched", or negative pressure, state. If one continues to decrease the coverage, however, the phase separated state will eventually become the favored state, and the "stretched" state will "snap", forming a droplet plus vacuum. For a simulation with a constant number of particles and variable area, this occurs when the derivative of the spreading pressure, $P=\rho^{2}(\partial e(\rho) / \partial \rho)$, is zero. At the spinodal point, the sound velocity becomes imaginary and the compressibility diverges. From the equation of state, we determine that this occurs at 0.034 atom $/ \AA^{2}$. For comparison, the spinodal density has been calculated to be between 031 and 0.038 atom $/ \AA^{2}$ for two-dimensional helium. 343332

From the polynomial fits for the two layers, we can estimate the regions of phase coexistence for the two solids by using the Maxwell double tangent construction. See Fig. 3. Since we have a constant number of particles and a variable density, the Maxwell construction is found from the common tangent of the energy per particle versus atomic area (inverse coverage). We determine the liquid-solid coexistence region to be from 0.0675 to 0.0700 atom $/ \AA^{2}$. This is comparahle to the ranges found for both two-dimensional helium 32,35 and the helium film,2 although the onset of full solidification that we find is at a somewhat lower density. The solidification density determined in this manner is subject to error, since our fitted parameters are not determined with a high degree of precision.

\section{B. Other properties}

As we have seen, in the absence of corrugations, the first layer has liquid-gas, self-bound liquid, liquid-solid, and solid regions. The gas phase has zero density at zero temperature. We expect the liquid-gas phase to have two 


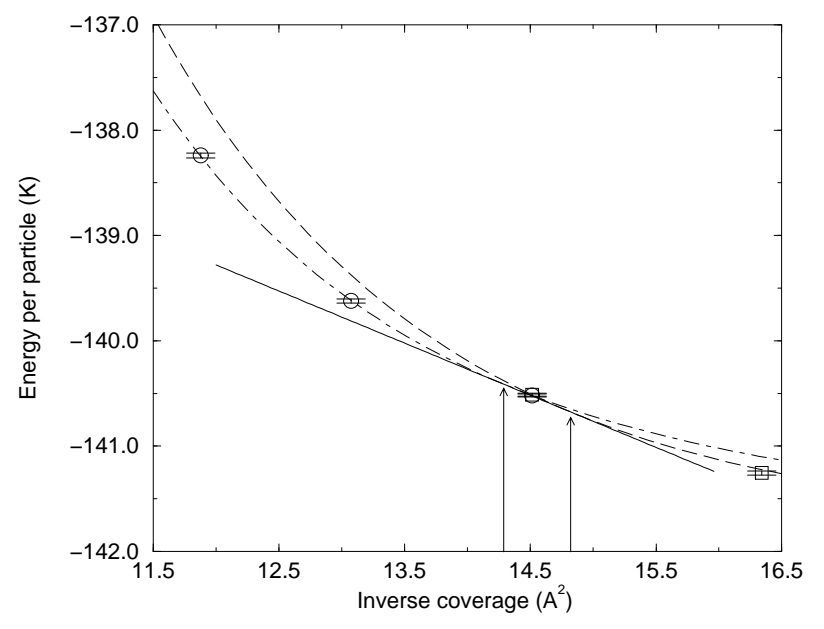

FIG. 3. Liquid-solid coexistence regions determined with the Maxwell construction. The dashed-dotted and dashed lines are the solid and liquid equations of state, respectively. Circles with error bars are calculated energy values for the liquid. Squares with error bars show values calculated for the solid phase. The unbroken line is the coexistence line. The arrows indicate the beginning and end of the coexistence region.

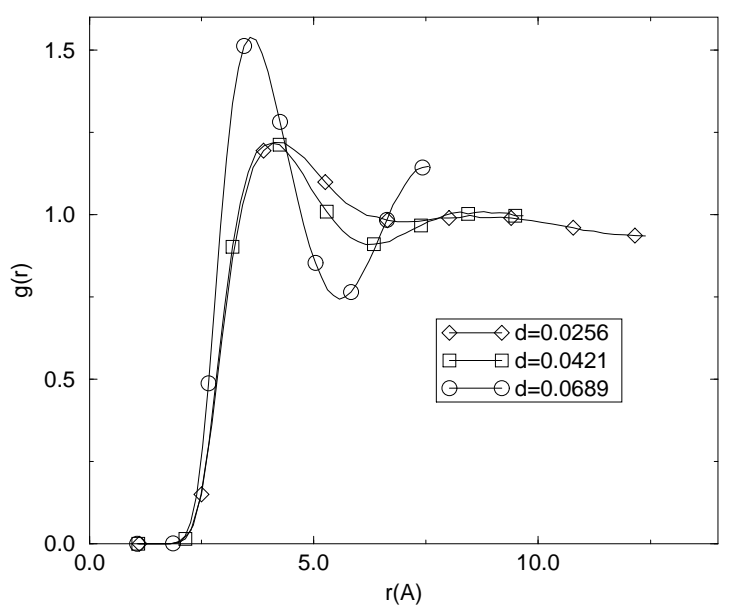

FIG. 4. Radial distribution function for the liquid phase at the indicated densities, in atom $/ \AA^{2}$.
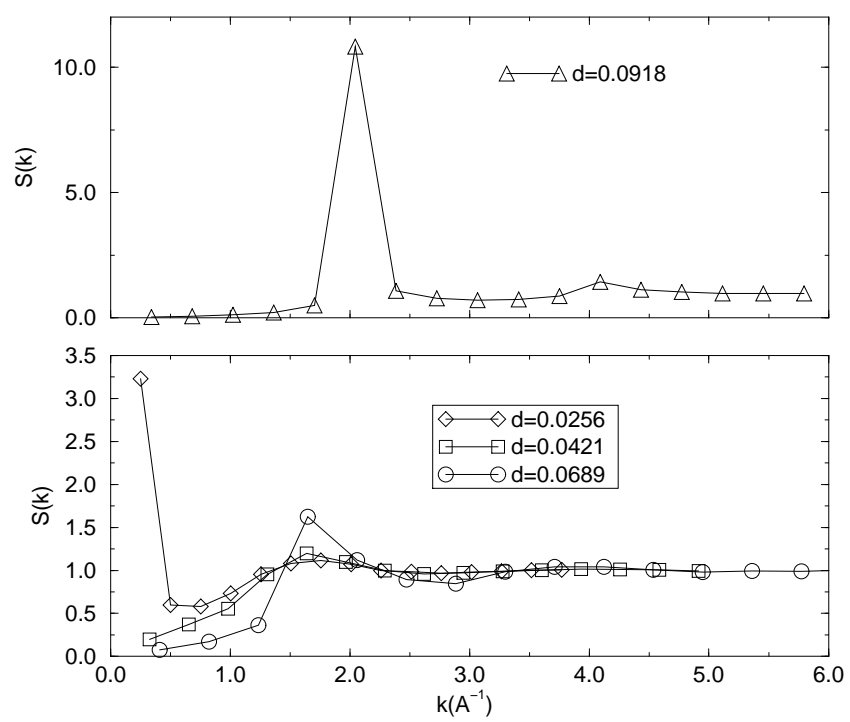

FIG. 5. Static structure function for the liquid phase at the indicated densities, in atom $/ \AA^{2}$. The static structure function in the (01) direction at a typical solid density (top) is also shown.
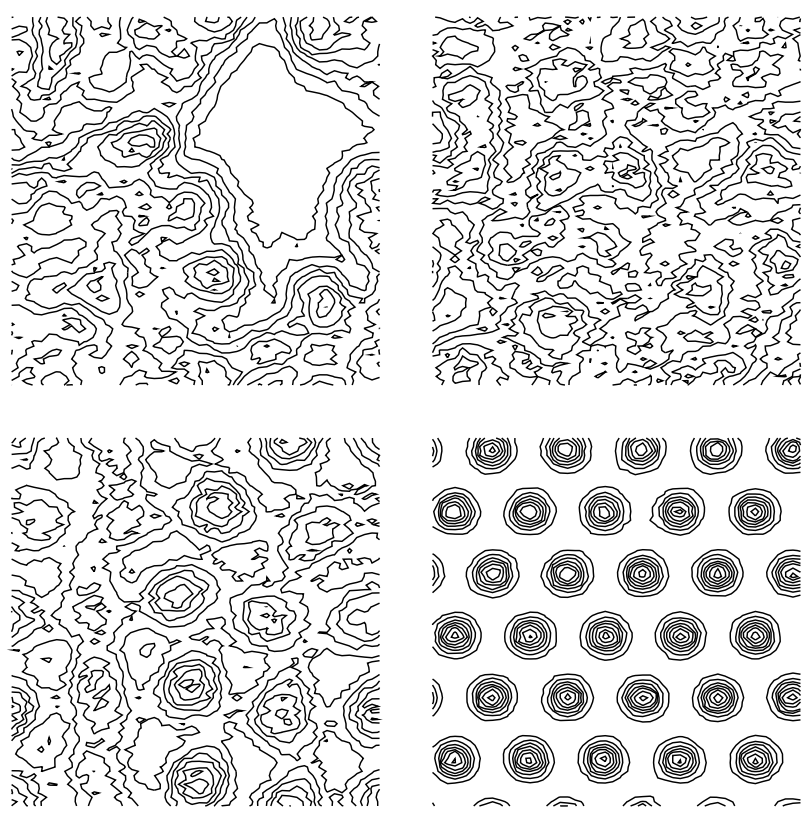

FIG. 6. Contour plots of the probability density in the plane of the substrate. The densities shown are, top row, left to right, 0.0256 and 0.0421 atom $/ \AA^{2}$; bottom row, left to right, 0.0689 , and 0.0918 atom $/ \AA^{2}$. 


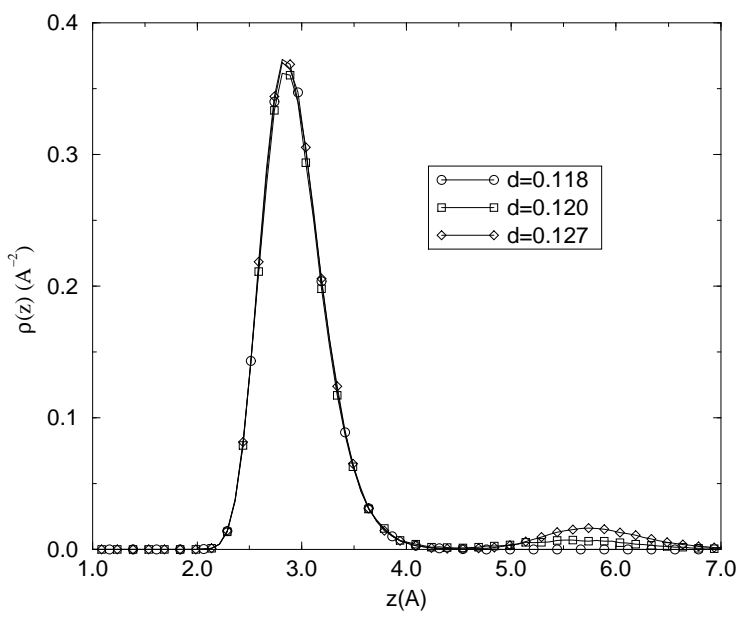

FIG. 7. Density profiles near second layer promotion. The coverages, in atom $/ \AA^{2}$, are indicated. Normalization is chosen so that integrating the profiles gives the coverage.

regions, one in which phase boundaries between these phases form, and one in which the liquid phase is artificially stretched. This is governed by the energy cost required to create the phase boundary. The metastable liquid phase is spatially indistinguishable from the equilibrium state, but the formation of droplets can be visualized. In Fig. 1 we plot the radial distribution function $\mathrm{g}(\mathrm{r})$ for the liquid at coverages in the droplet region, near equilibrium, and near the onset of liquid-solid coexistence. Here, $r$ is the magnitude of the projection of the distance vector between two particles onto the plane of the substrate. In the droplet region, the long range tail drops below unity, indicating the system does not uniformly cover the substrate. Near equilibrium the first peak has about the same height as the droplet phase, but the tail goes to unity at long ranges. The peaks are located at the same value of $r$, indicating that the average separation distance of nearest neighbor particles in the droplet is close to the equilibrium value. The high density liquid has noticeably more correlation. The peak height is 1.5 , compared to 1.2 near equilibrium, and the peak's position has shifted from 4.2 to $3.6 \stackrel{\AA}{A}$, as one would expect from compression caused by increasing the density. These values are in agreement with values reported for the helium liquid in two dimensions, 32 indicating that the first layer liquid is very two-dimensional in character.

The results for the static structure factor $S(k)$ for the

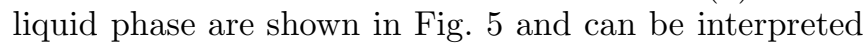
similarly. At the lowest coverage, $S(k)$ swings upward for low values of $k$ instead of going to zero. This indicates the presence of droplets and a nonzero compressibility. For the two uniform liquid coverages, $S(k \rightarrow 0) \rightarrow 0$. The structure function is more peaked for the high density liquid. Figure 5 also shows results for a typical solid coverage. The peak heights for the fluid near equilib- rium and for the dense fluid are 1.2 and 1.6, respectively. These values are in reasonable agreement with, but somewhat below, the two-dimensional values.

Finally, we show in Fig. 6 probability contours for the first layer for the various regions discussed above. The lowest coverage shown is below the spinodal point, and as expected incompletely covers the substrate. Near the equilibrium density, the substrate is uniformly covered. In the dense liquid, localization can be observed. This coverage is in the liquid-solid coexistence region. Finally the system enters a triangular solid phase at the highest density.

Promotion to the second layer may be determined by examining the density profiles for the dense solid. These are shown in Fig. I at the indicated coverages. The occupation of the second layer is clearly visible at the coverages 0.1200 and 0.1270 atom $/ \AA^{2}$, while the coverage 0.1180 shows no evidence of promotion. Integrating the profiles for the two largest coverages to the minimum between the peaks $(4.5 \AA)$ gives 0.116 and 0.119 atom $/ \AA^{2}$ for the first layer coverage. Experimentally, estimates 5 . 36. 37 国 of first layer completion range from 0.112 to 0.120 atom $/ \AA^{2}$ so our value is in good agreement. The recent GFMC calculation 24 obtain a value between 0.115 and 0.118 for the beginning of second layer promotion.

\section{RESULTS WITH CORRUGATED SUBSTRATE}

In this section we present results obtained by using a more realistic model of the graphite substrate. Most of the results shown in this section were obtained with a simulation cell with the dimensions $25.56 \AA \times 22.14 \AA$. The commensurate density corresponds to 36 helium atoms. Periodic boundary conditions were used in the plane of the substrate. Finite-size effects were examined by repeating some calculations using a $34.08 \AA \times 29.51 \AA$ simulation cell, for which the commensurate density corresponds to 64 particles.

\section{A. At And Below Commensurate Density}

The principal conclusion we obtain from these calculations is that the low density, low temperature phase of the first layer consists of commensurate solid clusters, rather than a liquid phase. In an earlier publication 22 we observed the commensurate phase and investigated its melting with both static structure and specific heat calculations. The calculated $\sqrt{3} \times \sqrt{3}$ commensurate solid phase and its melt are shown in Fig. 8. At $3 \mathrm{~K}$, the film has solidified into the commensurate structure. The solid forms a sub-lattice that contains one-third of the adsorption sites. The remaining two-thirds of the sites form two equivalent sub-lattices that are unoccupied. Raising the 

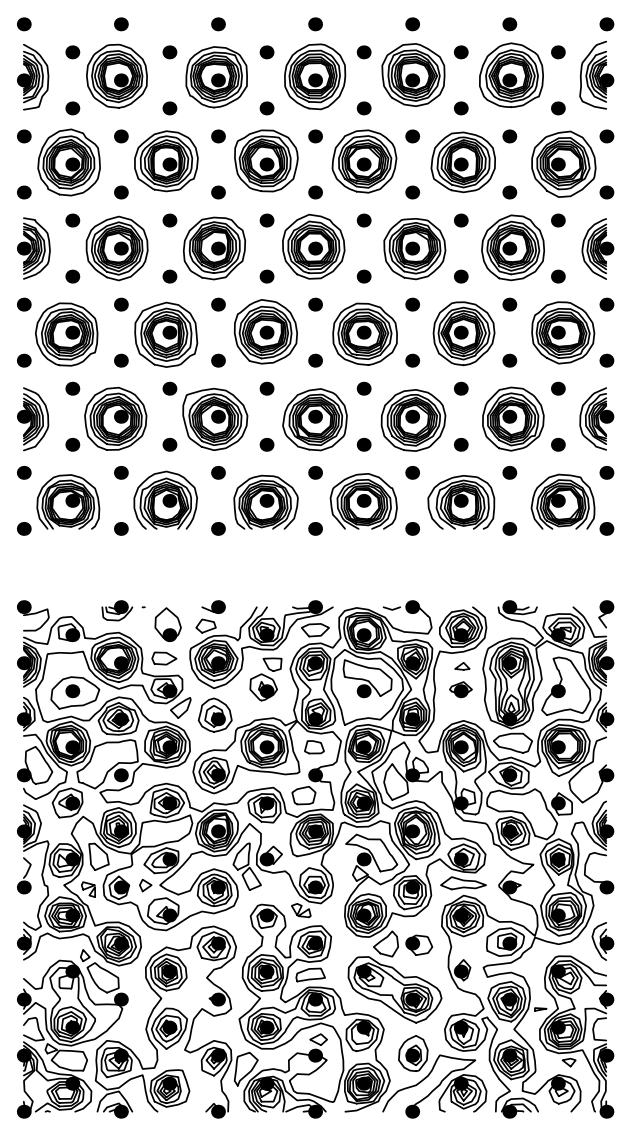

FIG. 8. Distribution plot at the commensurate density, 0.0636 atom $/ \AA^{2}$, for $\mathrm{T}=2.99 \mathrm{~K}$ and $\mathrm{T}=4.0 \mathrm{~K}$. The filled circles indicate graphite adsorption sites.

temperature to $4 \mathrm{~K}$ causes melting. At this temperature the helium atoms no longer inhabit a single sub-lattice of substrate adsorption sites. All adsorption sites will be occupied with equal probability if the simulation is run for a sufficiently long time.

Next, we present direct evidence that the low density (below the commensurate solid density of 0.0634 atom $/ \stackrel{\circ}{A}^{2}$ ) first layer consists of a solid cluster surrounded by a low density vapor at low temperatures. No liquid phase forms and so there is no possibility for first layer superfluidity. These findings are in contrast to the most recent proposal for this regions.

The presence of a solid with vacancies and phase separation can be visualized with contour plots of the probability distributions, shown in Fig. 9 for the indicated coverage and temperatures. At the lower temperature, $1.0 \mathrm{~K}$, the vacancies have condensed into a single bubble region, as can be seen in the top plot of Fig. 9. Note that periodic boundary conditions are being used, so the vacancy regions in the figure are connected. The vacancies move very slowly at this temperature; we found equilibration times to be very long for the vacancies to condense if they were initially spread throughout the lattice. We thus calculated the energy for this system twice: first
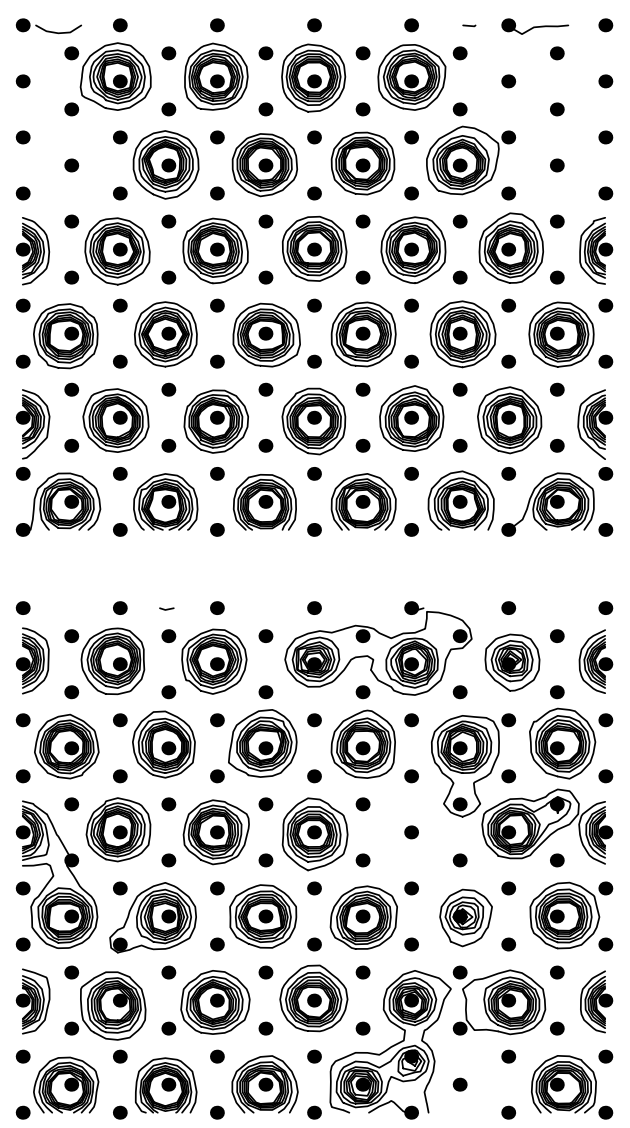

FIG. 9. Probability distributions for 0.0566 atom $/ \AA^{2}$ at $\mathrm{T}=1.0$ (top) and $2.5 \mathrm{~K}$ (bottom). The filled circles give the locations of graphite potential minima.

with the vacancies initially spread through the solid and then with the vacancies condensed. The condensed energy was found to be lower. At higher temperatures, the vacancies acquire enough kinetic energy to leave the phase separated state and diffuse into the solid. As a result, vacancies can become isolated. This is illustrated at $2.5 \mathrm{~K}$ in the lower plot of Fig. 9. A series of probability distribution plots reveals that these vacancies move in the simulation, so the equilibration problem encountered at $1.0 \mathrm{~K}$ is not present at this temperature. We note that we still see evidence of phase separation in contour plots at $2.0 \mathrm{~K}$, while heat capacity peaks from experiment seem to indicate a transition at $1.5 \mathrm{~K}$.

Figures 10 and 11 further confirm that the low density region contains solid clusters at low temperatures and that these clusters exhibit the melting behavior discussed in the previous section. We have calculated distribution plots for densities as low as 0.0207 atom $/ \AA^{2}$ and observe solid clusters at all densities.

We have also attempted to place a vacancy in a solid cluster to see if the cluster could support an isolated vacancy and at the same time be in equilibrium with the low density vapor. We found that at 0.0424 atom $/ \AA^{2}$ and $1.0 \mathrm{~K}$, the vacancy was spontaneously expelled from the 

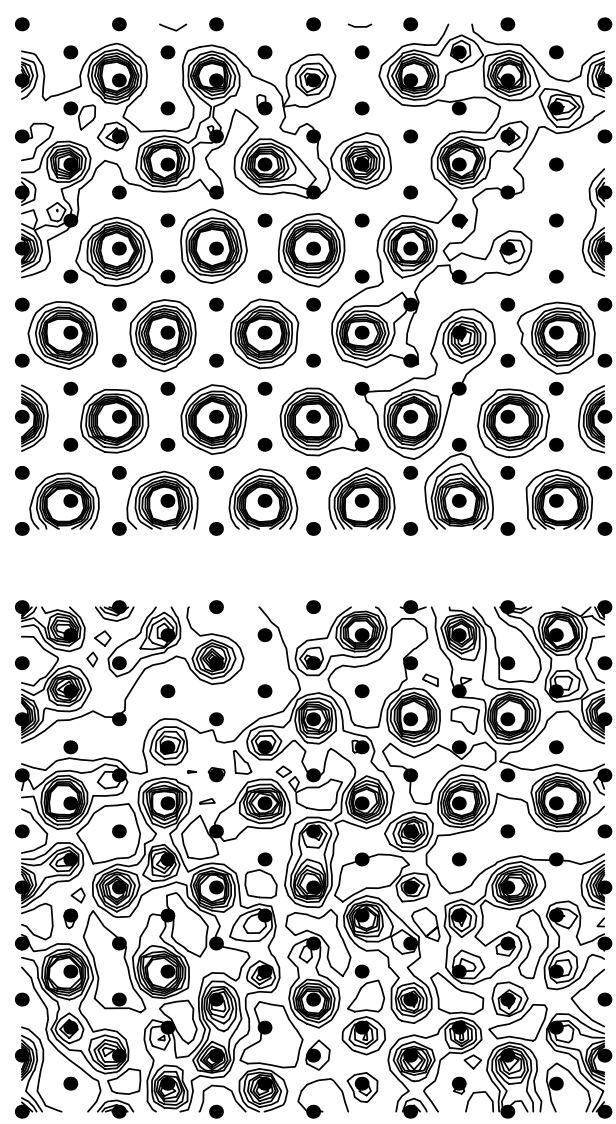

FIG. 10. Distribution plot at 0.0530 atom $/ \AA^{2}, \mathrm{~T}=2.5 \mathrm{~K}$ and $\mathrm{T}=3.0 \mathrm{~K}$

solid cluster during thermalization. We conclude that at low temperatures the solid clusters will not support isolated vacancies.

Further evidence for solidification into the commensurate structure in the simulation comes from calculations of the static structure factor. Typical results for static structure factors for coverages at and immediately below the commensurate solid density have been reported in our earlier paper22. In addition, the melting of the commensurate solid phase can be determined from the temperature dependence of the static structure peak height. Melting is signaled by a significant drop in the peak height and a large statistical fluctuation in a peak value near the melting temperatures. The melting temperature determined in this manner are given in Table II. The density dependence of the melting temperature is consistent with the experimental phase diagram, although our melting temperatures are slightly higher than the experimental values. Heat capacity measurements indicate that the commensurate solid melts at about $3 \mathrm{~K}$, and the low density $\left(<0.045\right.$ atom $\left./ \AA^{2}\right)$ melting peaks are at about $1.5 \mathrm{~K}$.

Another way of estimating melting temperatures is from the temperature dependence of the energy. This is shown in Fig. 12 for various densities. These curves
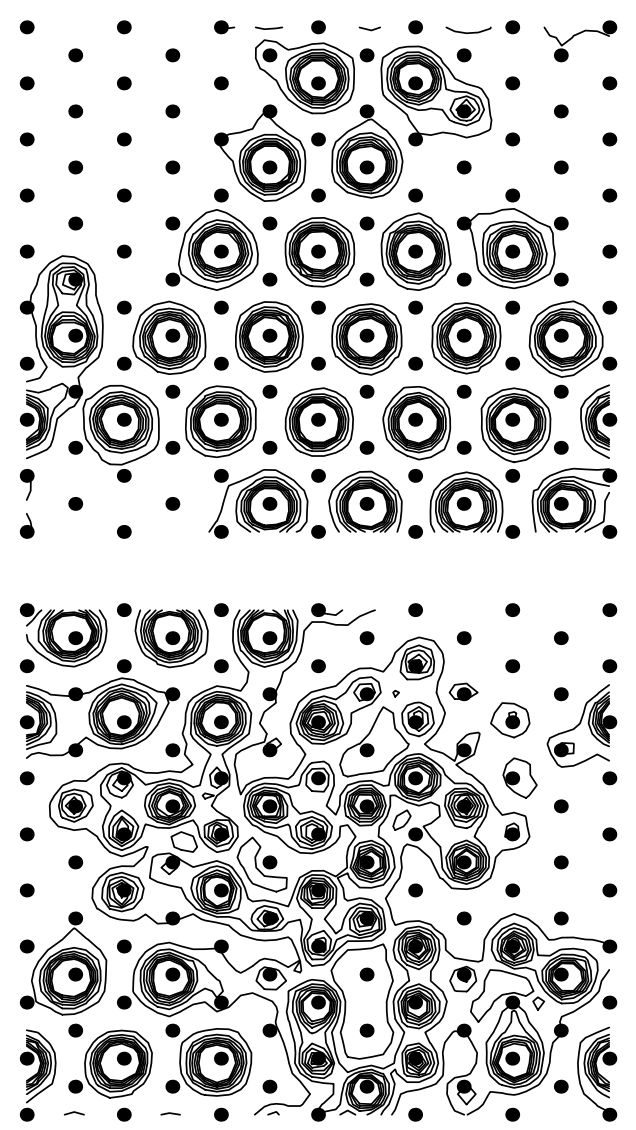

FIG. 11. Distribution plot at 0.0424 atom $/ \AA^{2}, T=1.0 \mathrm{~K}$ and $\mathrm{T}=2.0 \mathrm{~K}$

possess inflection points that lead to specific heat maxima when differentiated. These peaks indicate melting. Figure 13 shows two sample calculations of the specific heat at the indicated densities. The peak height and location change dramatically with density. Melting occurs at about $1.5 \mathrm{~K}$ and $3.5 \mathrm{~K}$ for 0.0353 (filled circles) and 0.0636 atom $/ \AA^{2}$ (open squares) respectively. In Fig. 13 the calculated specific heat for 0.0353 and 0.0636 atoms $/ \AA^{2}$ is shown. For comparison we also present the experimental measure specific heat (solid lines) for densities of 0.0367 and 0.0634 atoms $/ A A^{2}$. The calculated specific heat has peaks somewhat above the experimental values but are consistent with the melting behavior exhibited in the contour plots and in the static structure factor calculations.

\section{B. Maxwell Construction}

We determine phase ranges by applying the Maxwell construction to the total ground state energy. For a system at constant volume with a varying number of particles, a region of phase separation will be signaled by an unphysical upward curvature in the total free energy's dependence on density. The upper and lower bounding 
TABLE II. Estimates of melting temperatures from the temperature dependence of the static structure peaks.

\begin{tabular}{|c|c|}
\hline \hline Coverage (atom $\left./ \AA^{2}\right)$ & $\mathrm{T}_{\text {melt }}$ \\
\hline 0.0424 & 2.0 \\
0.0530 & 2.5 \\
0.0566 & 3.0 \\
0.0636 & 3.33 \\
\hline \hline
\end{tabular}

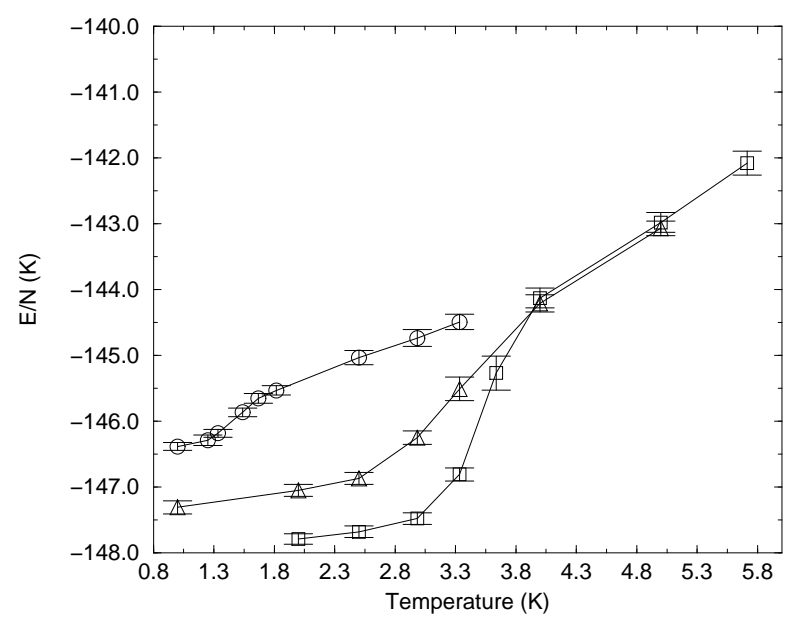

FIG. 12. Temperature dependence of the energy per particle. The coverages are 0.0353 (circles), 0.0566 (triangles), and 0.0636 atom $/ \AA^{2}$ (squares).

densities of this region are connected by a common tangent line. The total free energy for all intermediate densities lies on or above this line, either because creating a surface between the two phases costs a finite amount of energy or because the system remains unphysically homogeneous. In the thermodynamic limit the system will separate into the two phases at the bounding densities.

The Maxwell construction at nonzero temperatures should be applied to the total free energy. This is not directly accessible from PIMC, however. Instead, we use a limiting process to determine effectively ground state energy values. All energy calculations are performed at low temperatures. The temperature is then raised and the energy is recalculated. If the two values are the same within error bars, we conclude that we have obtained effectively zero temperature energy values. This allows us to apply the Maxwell construction to the total energy, since it is the same as the total free energy at zero temperature.

We now can apply this procedure to the total energy calculations of the first layer solid. Total energy calculations for a range of densities using a simulation cell designed to accommodate the commensurate solid are

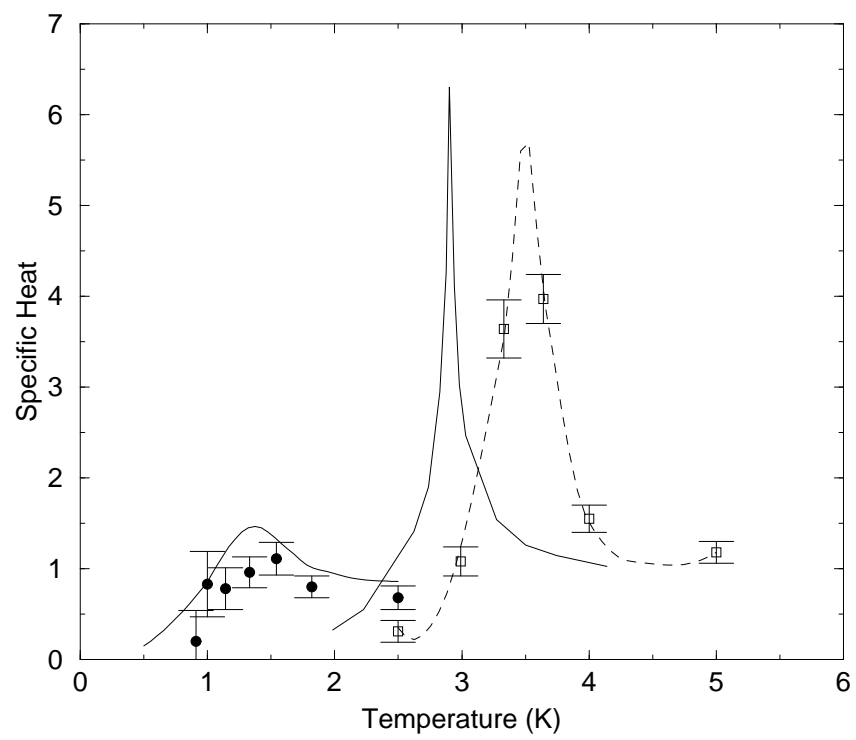

FIG. 13. Specific heat for 0.0353 (filled circles) and 0.0636 atom $/ \AA^{2}$ (squares). The dashed line is a guide to the eye. The solid lines are the experimentally determined specific heat at 0.0367 and 0.0634 atom $/ \AA^{2}$.

TABLE III. Energy/particle versus coverage. The first column gives the temperature of the calculation. The number in parenthesis gives the error in the last decimal place.

\begin{tabular}{|c|c|r|}
\hline \hline $\mathrm{T}(\mathrm{K})$ & $\sigma\left(\mathscr{A}^{-2}\right)$ & $\mathrm{E} / \mathrm{N}(\mathrm{K})$ \\
\hline 1.00 & 0.0353 & $-143.73(8)$ \\
1.00 & 0.0424 & $-144.03(9)$ \\
1.00 & 0.0530 & $-144.31(7)$ \\
1.00 & 0.0566 & $-144.56(7)$ \\
1.00 & 0.0636 & $-145.12(8)$ \\
1.33 & 0.0707 & $-142.71(8)$ \\
\hline \hline
\end{tabular}

shown in Fig. 14. The values for the energy per particle are given in Table III. The cell dimensions are $25.560 \AA \times 22.136 \AA$, and the number of particles varies from 20 to 40 . The dashed line in Fig. 14 is a straight line connecting the lowest density accessible in our lattice and the density that corresponds to $1 / 3$ coverage. Notice that all intermediate energy values are above this straight line. From the Maxwell construction, this indicates that the intermediate energy values are unstable and will phase separate into the two stable phases (the vapor and the commensurate solid) that bound the unstable region.

The binding energy for a single particle on the substrate can be easily calculated. We find this to be $E_{B}=$ $-143.29 \pm 0.27$. This is comparable to the estimated values $38 E_{B}=-141.75 \pm 1.50 \mathrm{~K}$ from scattering 39.40 and $E_{B}=-142.33 \pm 1.97 \mathrm{~K}$ from thermodynamic analysis 41 . Our value for the binding energy was calculated at 0.4 $\mathrm{K}$ and confirmed to be the ground state value by the 


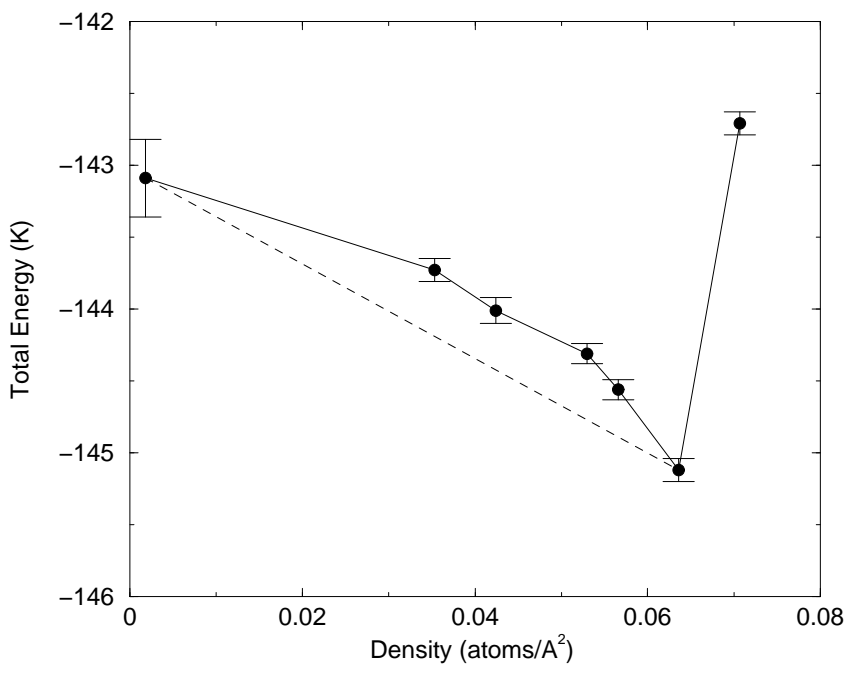

FIG. 14. Total energy versus coverage. The dashed line is a straight line connecting the lowest density accessible in our lattice and the density that corresponds to $1 / 3$ coverage.

limiting procedure discussed above. By subtracting this energy from the energy per particle of the commensurate solid phase, we obtain the condensation energy per particle for the two-dimensional solid, $E_{2 D}=-2.03 \pm 0.20$ $\mathrm{K}$. This is comparable to, but slightly higher than, the two-dimensional energy $(-1.06 \mathrm{~K})$ for the commensurate phase found by the variational calculations ofd for the same interaction model.

\section{Above Commensurate Density}

Figures 15 and 16 illustrate the domain wall solid and incommensurate triangular solid phases, respectively. The domain wall solid consists of patches of commensurate solid on different sub-lattices. Linear domain walls occur at the boundaries between these regions. In the incommensurate solid, the atoms form a triangular solid that does not have a periodic relationship with the underlying adsorption sites for lengths scales less than the minimum dimension of the simulation cell.

\section{TESTS OF OUR CONCLUSIONS}

The strength of our conclusions is limited by the accuracy of the interaction model that we use. First, it is possible that the substrate may substantially alter the helium-helium interaction 39 .29. Inclusion of this effect, the so-called McLachlan interaction, has been shown to change the ground state phase from the commensurate solid to a low density liquid in two-dimensional variational calculations.20 We have repeated the low temperature density scans using the same mediated interactions employed in these calculations 20 . We found that while the energy per particle increases for all coverages,

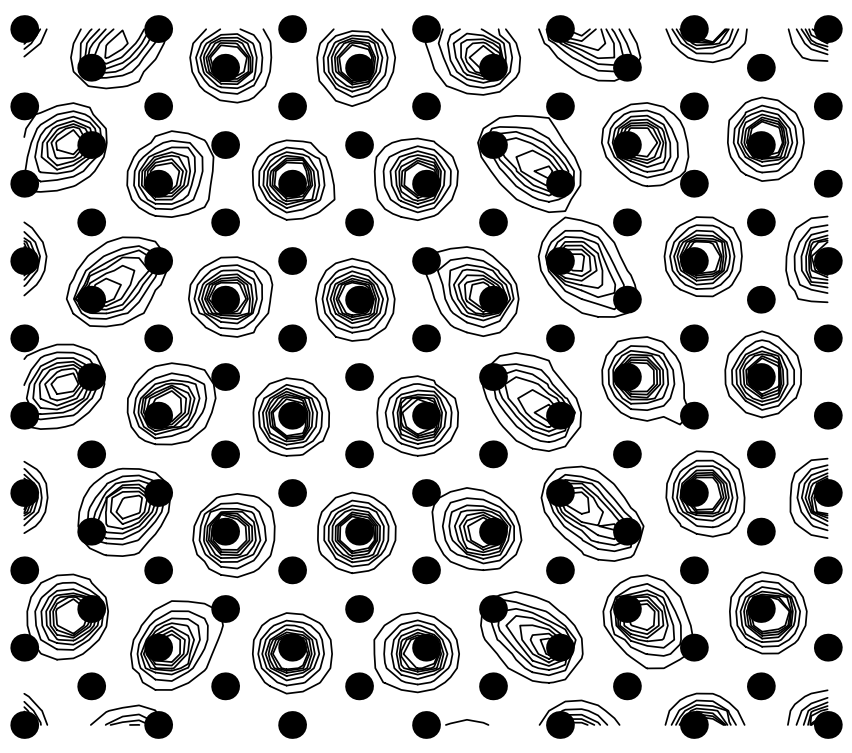

FIG. 15. Distribution plot of the domain wall solid at 0.0742 atom $/ \AA^{2}(\mathrm{~N}=42$ atoms $)$ and $\mathrm{T}=1.0 \mathrm{~K}$. Filled circles indicate adsorption sites.

the commensurate solid remains the energetically favored phase. Second, another petential problem is that the helium-graphite interaction 23 is too corrugated, thus favoring solidification. We have repeated the low temperature calculations at the commensurate density and at 0.0424 atom $/ \AA^{2}$ with the corrugation strength reduced by $25 \%$. The commensurate phase remains energetically favored. Calculations were also performed with the corrugation reduced by $50 \%$, but it was found that the commensurate solid would not form for temperatures as low as $2 \mathrm{~K}$, thus indicating the corrugations had been underestimated. We note finally that the melting behavior of the commensurate solid phase was not sensitive to the inclusion of the McLachlan term.

Finally, we wish to discuss the arguments of Greywall and Busch (GB) against solid clusters and in favor of the superfluid phase. Their primary objection to solid-vapor coexistence is that this should be signaled by linear heat capacity isotherms for the entire region from zero coverage up to the commensurate density. Their published data shows that for temperatures from $0.2 \mathrm{~K}$ to $0.5 \mathrm{~K}$, the isotherms are linear only between 0.025 and 0.060 atom $/ \AA^{2}$. At $0.1 \mathrm{~K}$, the upper endpoint is about 0.055 atom $/ \AA^{2}$. As a possible explanation, we suggest that the 


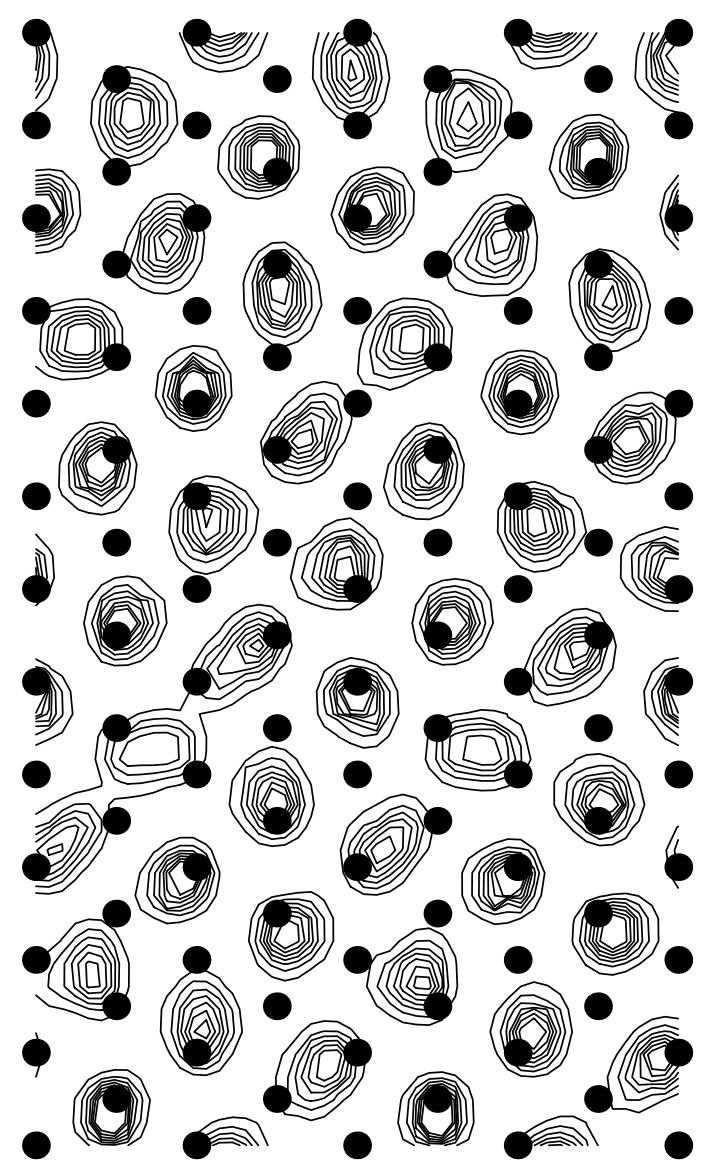

FIG. 16. Distribution plot of the incommensurate solid phase at 0.0994 atom $/ \AA^{2}(\mathrm{~N}=50$ atoms $)$ and $\mathrm{T}=2.0$. Filled circles indicate adsorption sites.

departure from linearity below 0.025 atom/ $/ \AA^{2}$ is caused by the presence of multiple finite-sized clusters. At low densities, solid clusters nucleate around surface defects. Initially, there are many small metastable clusters with large perimeter-to-area ratios. Increasing the density increases the size of the clusters until the surface is covered by a few large solid clusters with negligible boundary effects. Thus, the heat capacity exhibits linear behavior only after the solid clusters are sufficiently large so that the perimeter-to-area ratio is small. This presumably occurs for coverages above 0.025 atom $/ \AA^{2}$. GB have used a similar explanation in their arguments for solid-liquid and liquid-gas coexistence in regions that do not have linear isotherms.

GB's identification of coverages near 0.04 atom $/ \AA^{2}$ as liquid is based partly on simulation results for $2 \mathrm{D}$ helium on a flat substrate, the most relevant calculations then available. As GB note, the large peak associated with the melting of the uniform commensurate solid phase first emerges above 0.04 atom $/ \AA^{2}$. 2D helium is a liquid near this density 32 , suggesting that first layer coverages below 0.04 may be liquid. Unlike the purely $2 \mathrm{D}$ simulations, our calculations take the role of substrate effects into account. As we have shown, surface corrugations push the density of the energy minimum up from about 0.04 on a flat substrate to 0.0636 atom $/ \AA^{2}$ and produce solidification. GB also show that their low density heat capacity results are in general agreement with a PIMC calculation for $2 \mathrm{D}$ superfluid helium 42 , suggesting that there might be a superfluid transition in the first layer. We have shown in Fig. 13 that these rounded heat capacities are produced by the melting of a solid cluster and are not associated with a superfluid transition.

In closing, we would like to remark that a promising direction for monolayer superfluidity 5.435 lies with helium adsorbed on alkali substrates, particularly lithium 46.44 . These substrates have much smaller corrugations and a much weaker attraction, allowing the first layer helium film to be a liquid. The phenomenon competing with superfluidity for these substrates is pre-wetting, rather than solidification.

\section{SUMMARY}

Our first layer calculations were performed both with and without substrate corrugations. When corrugations are neglected, the first layer film resembles a purely twodimensional film. We determined that the film consists of gas, liquid, and solid phases. These are separated by coexistence regions, and we determined the coverage ranges for all phases at low temperatures by using the Maxwell construction. The first layer liquid has an equilibrium density of 0.0450 atoms $/ \AA^{2}$. Below this density the system phase separates. This region is divided into an unstable region, where liquid droplets form, and a metastable region in which the system over-expands instead of forming an interface. These two regions are separated by a spinodal point at 0.034 atoms $/ \AA^{2}$. At higher densities the system enters a narrow region of liquid-solid coexistence between 0.0675 and 0.0700 atoms $/ \AA^{2}$. Above these coverages the system is in a triangular solid phase. The beginning of layer promotion occurs between 0.116 and 0.119 atoms $/ \AA^{2}$. All of our calculations are in agreement with recent Green's function Monte Carlo results.24

When corrugations are included in the first layer, the phase diagram is substantially altered. We find that a $\sqrt{3} \times \sqrt{3}$ commensurate solid occurs, in agreement with numerous experiments. By examining the temperature dependence of the static structure function and the specific heat, we find that this solid melts at approximately $3.5 \mathrm{~K}$, compared with the experimental melting temperature of $3 \mathrm{~K}$. We further find that the commensurate solid phase is energetically favored. At densities below commensuration, the system phase separates into commensurate solid clusters and a low density vapor. No liquid phase occurs at low temperatures. 


\section{ACKNOWLEDGMENTS}

This work was supported in part by the National Aeronautics and Space Administration under grant number NAG3-1841. Some of the calculations were performed using the facilities of the Supercomputer Computations Research Institute at Florida State University. We also wish to thank L. W. Bruch for useful comments on our work.

${ }^{1}$ J. G. Dash, Films on Solid Surfaces (Academic, New York, 1975).

${ }^{2}$ M. Schick, in Phase Transitions in Surface Films, edited by J. G. Dash and J. Ruvalds (Plenum, New York, 1980).

${ }^{3}$ L. W. Bruch, M. W. Cole, and E. Zaremba, Physical Adsorption: Forces and Phenomena (Oxford, New York, 1997).

${ }^{4}$ M. Bretz, Phys. Rev. Lett. 38, 501 (1977).

${ }^{5}$ D. S. Greywall and P. A. Busch, Phys. Rev. Lett. 67, 3535 (1991).

${ }^{6}$ D. S. Greywall, Phys. Rev. B 47, 309 (1993).

${ }^{7}$ P. A. Crowell and J. D. Reppy, Physica B 187, 278 (1994).

${ }^{8}$ P. A. Crowell and J. D. Reppy, Phys. Rev. B 53, 2701 (1996).

${ }^{9}$ M. Nielsen, J. P. McTague, and L. Passell, in Phase Transitions in Surface Films, edited by J. G. Dash and J. Ruvalds (Plenum, New York, 1980).

${ }^{10}$ F. F. Abraham and J. Q. Broughton, Phys. Rev. Lett. 59, 64 (1987).

${ }^{11}$ K. Carneiro et al., Phys. Rev. Lett. 37, 1695 (1976).

12 S. V. Hering, S. W. V. Sciver, and O. E. Vilches, J. Low Temp. Phys. 25, 793 (1976).

13 T. A. Rabedeau, Phys. Rev. B 39, 9643 (1989).

${ }^{14}$ R. E. Ecke, Q.-S. Shu, T. S. Sullivan, and O. E. Vilches, Phys. Rev. B 31, 448 (1985).

${ }^{15}$ M. Bretz, Phys. Rev. Lett. 31, 1447 (1973).

16 A. N. Berker, S. Ostlund, and F. A. Putnam, Phys. Rev. B 17, 3650 (1978).

${ }^{17}$ M. Schick, J. S. Walker, and M. Wortis, Phys. Rev. B 16, 2205 (1977).

${ }^{18}$ M. J. Tejwani, O. Ferrerira, and O. E. Vilches, Phys. Rev. Lett. 44, 152 (1980).

19 S. B. Crary and D. A. Fahey, Phys. Rev. B 35, 2102 (1987).

${ }^{20}$ J. M. Gottlieb and L. W. Bruch, Phys. Rev. B 48, 3943 (1993).

${ }^{21}$ P. Mohandas et al., J. Low Temp. Phys. 101, 481 (1995).

${ }^{22}$ M. Pierce and E. Manousakis, editorially approved for publication in Phys. Rev. Lett.

${ }^{23}$ W. E. Carlos and M. W. Cole, Surf. Sci. 91, 339 (1980).

${ }^{24}$ P. A. Whitlock, G. V. Chester, and B. Krishnamachari, Phys. Rev. B 58, 8704 (1998).

${ }^{25}$ D. M. Ceperley, Rev. Mod. Phys. 67, 279 (1995).

${ }^{26}$ M. Pierce and E. Manousakis, Phys. Rev. B 59, 3802 (1999) and Phys. Rev. Lett. 81, 156 (1998).
${ }^{27}$ M. Takahashi and M. Imada, J. Phys. Soc. Jpn 53, 3765 (1984).

${ }^{28}$ R. A. Aziz et al., Mol. Phys. 77, 321 (1992).

${ }^{29}$ L. W. Bruch, Surf. Sci. 125, 194 (1982).

30 A. D. McLachlan, Mol. Phys. 7, 381 (1964).

${ }^{31}$ R. A. Aziz et al., J. Chem. Phys. 70, 4330 (1979).

${ }^{32}$ P. A. Whitlock, G. V. Chester, and M. H. Kalos, Phys. Rev. B 38, 2418 (1988).

${ }^{33}$ S. Giorgini, J. Boronat, and J. Casulleras, Phys. Rev. B 54, 6099 (1996).

${ }^{34}$ B. E. Clements, J. L. Epstein, E. Krotcheck, and M. Saarela, Phys. Rev. B 48, 7450 (1993).

${ }^{35}$ M. C. Gordillo and D. M. Ceperley, Phys. Rev. B 58, 6447 (1998).

${ }^{36}$ S. E. Polanco and M. Bretz, Phys. Rev. B 17, 151 (1978).

${ }^{37}$ K. Carneiro, L. Passell, W. Thomlinson, and H. Taub, Phys. Rev. B 24, 1170 (1981).

${ }^{38}$ M. W. Cole and D. L. Goodstein, Rev. Mod. Phys. 53, 199 (1981).

${ }^{39}$ G. D. Derry, D. Wesner, W. E. Carlos, and D. R. Frankl, Surf. Sci. 87, 629 (1979).

${ }^{40}$ W. E. Carlos and M. W. Cole, Phys. Rev. B 21, 3713 (1980).

${ }^{41}$ R. L. Elgin and L. Goodstein, Phys. Rev. A 9, 2657 (1974).

${ }^{42}$ D. M. Ceperley and E. L. Pollock, Phys. Rev. B 39, 2084 (1989).

43 J. M. Mochel and M.-T. Chen, Physica B 197, 278 (1994).

${ }^{44}$ M. Boninsegni and M. W. Cole, J. Low Temp. Phys. 113, 393 (1998).

45 J. Nyéki, R. Ray, B. Cowan, and J. Saunders, Phys. Rev. Lett. 81, 152 (1998).

${ }^{46}$ E. Cheng, G. Ihm, and M. W. Cole, J. Low Temp. Phys. 74, 519 (1989). 